\title{
A Measuring Theorem of the Resistivity Tensor Parameters of Thin Anisotropic Conductors by a Six-Probe Method
}

\author{
Member Hisashi Shibata (Ibaraki College of Technology) \\ Member Ryuiti Terakado (Fukushima College of Technology)
}

Key words : Anisotropic conductor, Resistivity measurement

The four probe methods ${ }^{(1)(2)}$ for measuring the resistivity of isotropic materials have widely been used as a standard one in semiconductor technology. For the method ${ }^{(2)}$ with the probes on the circumference of the flat sample of arbitrary shape, we have recently presented the general solution ${ }^{(3)}$ of an anisotropic conductor with an arbitrary resistivity tensor. The application of the method ${ }^{(1)}$ with the probes on the surface of the sample to the resistivity measurements of an anisotropic conductor has been presented by Wasscher ${ }^{(4)}$. However, his method requires the knowledge of the directions of the principal axes of the resistivity tensor. In this letter, a measuring theorem of the resistivity tensor parameters, the principal axes resistivities and the directions of principal axes, for thin anisotropic materials is proposed.

It is assumed that the material to be measured is a flat and thin sample of uniform thickness $w$ and is enough large compared to the probe distances. The probe arrangement and the circuit for the determination of the resistivity tensor parameters of an anisotropic material are shown in Fig. 1. Six sharp probes are placed on the surface of the material. The spacing between adjacent probes is an equal distance of s. In Fig. 1, the current $I$ is passed through the two probes designated as $A$ and $B$, and the potential differences $V_{i 0}(i=1,2,3)$ between the probe points of $i(=1,2,3)$ and 0 are measured, respectively.

We here present the determining method of the principal axes resistivities, $\rho_{\xi}$ and $\rho_{\eta}$, of the conductor and the angle $\theta$ between the $x$-axis and the $\xi$ axis. Suppose that the $x$-axis is parallel to the line

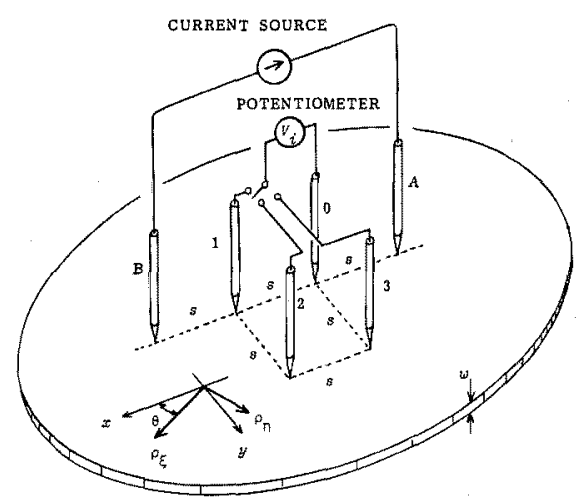

Fig. 1. Probe arrangements and the circuit for obtaining the resistivity tensor parameters of thin anisotropic materials.

connecting the two current probes. $\rho_{\xi}, \rho_{\eta}$ and $\theta$ can be calculated from the measured values of $V_{10}, V_{20}$ and $V_{30}$ as follows:

$$
\begin{aligned}
\rho_{\xi}= & \rho / 2 d\left[\left(b^{2}+d^{2}+1\right)\right. \\
& \left. \pm \sqrt{\left(b^{2}+d^{2}+1\right)^{2}-4 d^{2}}\right] . \\
\rho_{\eta}= & \rho / 2 d\left[\left(b^{2}+d^{2}+1\right)\right. \\
& \left.\mp \sqrt{\left(b^{2}+d^{2}+1\right)^{2}-4 d^{2}}\right] \\
\theta= & \tan ^{-1}\left[\left\{\left(b^{2}+d^{2}\right)-1\right.\right. \\
\mp & \left.\left.\sqrt{\left(b^{2}+d^{2}+1\right)^{2}-4 d^{2}}\right] / 2 b\right]
\end{aligned}
$$

where

$$
\begin{aligned}
& b=\frac{3(\alpha \beta-1)}{2(2 \alpha-4 \beta+\alpha \beta+1)} \\
& d=\sqrt{\frac{\alpha(1-b)^{2}-(2+b)^{2}}{1-\alpha}} . \\
& \alpha=\frac{1}{4} \exp \left(4 \ln 2 \frac{V_{20}}{V_{10}}\right) \cdots \cdots . .
\end{aligned}
$$




$$
\begin{aligned}
& \beta=\frac{1}{4} \exp \left(4 \ln 2 \frac{V_{30}}{V_{10}}\right) \cdots \\
& \rho=-(w \pi / \ln 2)\left(V_{10} / I\right)
\end{aligned}
$$

These equations can be derived by obtaining three equations for $V_{i 0}(i=1,2,3)$ in the equivalent isotropic region after applying Eq. (2) in Ref. (5) and by solving the equations for $\rho_{\xi}, \rho_{\eta}$ and $\theta$, for the case when the conductor in Fig. 1 is infinitely wide.

$\rho_{\xi}, \rho_{\eta}$ and $\theta$ in Eqs. ( 1$) \sim(3)$ have two values, respectively. This corresponds to the situations of $\rho_{\xi}, \rho_{\eta}$ and $\theta$ to be measured. The situations can be divided into the cases as:

$$
\left.\begin{array}{ll}
\text { (i) } \rho_{\xi}>\rho_{\eta} \text { and } 0 \leqq \theta<\pi / 2 \\
\text { (ii) } \rho_{\xi}<\rho_{\eta} \text { and } 0 \leqq \theta<\pi / 2 \\
\text { (iii) } \rho_{\xi}=\rho_{\eta}
\end{array}\right\}
$$

Therefore, the determination of the sign in Eqs. (1) $\sim(3)$ is needed. Now, let $\rho_{\xi}(+)$ and $\rho_{\xi}(-)$ be the values of $\rho_{\xi}$ for the plus sign and the minus sign in Eq. (1), respectively. Similarly, we use the expressions of $\rho_{\eta}(+), \rho_{\eta}(-), \theta(+)$ and $\theta(-)$ for the difference in signs in Eqs. (2) and (3).

The sign in Eqs. (1) and (2) can be determined by using the value of $b$ in Eq. (4) as:

$$
\begin{array}{r}
\rho_{\xi}=\rho_{\xi}(+), \rho_{\eta}=\rho_{\eta}(-), \rho_{\xi}>\rho_{\eta} \\
\quad(\text { when } b>0) \\
\rho_{\xi}=\rho_{\xi}(-), \rho_{\eta}=\rho_{\eta}(+), \rho_{\xi}<\rho_{\eta} \\
\quad(\text { when } b<0)
\end{array}
$$

because $b$ has an immediate connection with $\rho_{\xi}, \rho_{\eta}$ and $\theta$ as :

$$
b=\frac{\left(\rho_{\xi}-\rho_{\eta}\right) \sin \theta \cos \theta}{\rho_{\xi} \cos ^{2} \theta+\rho_{\eta} \sin ^{2} \theta}
$$

Eq. (12) can be obtained from Eqs. (1) ( 3 ). Furthermore, we determine the sign in eq. ( 3 ) as:

$$
\theta= \begin{cases}\theta(+) & \{\text { when } \theta(+)>0] \\ \theta(-) & \{\text { when } \theta(-)>0\}\end{cases}
$$

since $0 \leqq \theta<\pi / 2$ and $|\theta(+)-\theta(-)|=\pi / 2$.

For both cases of $\rho_{\xi}=\rho_{\eta}$ and $\theta=0$ in Eq. ( 9 ), it is clear from Eq. (12) that the value of $b$ is zero. Therefore, the classification into detailed cases is done by using the value of $d$ in Eq. ( 5 ) as :

$$
\begin{aligned}
& \rho_{\xi}=\rho_{\eta}=\rho \quad(\text { when } b=0 \text { and } d=1) \quad \cdots \cdots(14) \\
& \theta=0\left\{\begin{array}{c}
\rho_{\xi}=\rho / d, \quad \rho_{\eta}=d \rho, \quad \rho_{\xi}>\rho_{\eta} \\
\quad(\text { when } b=0 \text { and } d<1) \cdots \cdots \cdots(15) \\
\rho_{\xi}=\rho / d, \quad \rho_{\eta}=d \rho, \quad \rho_{\xi}<\rho_{\eta} \\
\quad(\text { when } b=0 \text { and } d>1) \cdots \cdots \cdots(16)
\end{array}\right.
\end{aligned}
$$

This judgment is based on the following relation which can be obtained from Eqs. ( 1$) \sim(3)$.

$$
d=\sqrt{\rho_{\xi} \rho_{\eta}} /\left(\rho_{\xi} \cos ^{2} \theta+\dot{\rho}_{\eta} \sin ^{2} \theta\right)
$$

There are many ways of arranging the four potential probes in addition to that of Fig. 1.

A pocket calculator is sufficient for obtaining the values of $\rho_{\xi}, \rho_{\eta}$ and $\theta$ from the measured values of the potential differences. However, since only infinite samples have been analyzed in this letter, it is necessary to make corrections in Eqs. ( 1$) \sim(3)$ for the finite dimensions of the samples.

(Manuscript received Dec. 25, 1989)

\section{References}

(1) L. B. Valdes: Proc, IRE, 42, 420 (1954)

(2) L. T. van der Pauw: Philips Res. Rept., 13, 1 (1958)

(3) H. Shibata \& R. Terakado, J. Appl. Phys., 66, 4603 (1989)

(4) J. D. Wasscher: Philips Res. Rept., 16, 301 (1961)

(.5) H. Shibata \& R. Terakado: Electronics Letters, 19, 518 (1983)

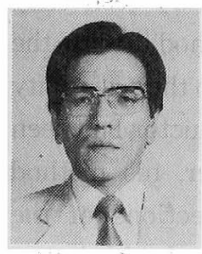

\section{Hisashi Shibata (Member)}

He was born in Ibaraki, Japan, on Feb. 5,1950 . He received the B. E. degree in electrical engineering from Ibaraki University, Ibaraki, Japan, in 1975. He joined the Department of Electrical Engineering, Ibaraki College of Technology, Katsuta, Ibaraki, Japan, in 1975, where he is now an Associate Professor. His research interest is the analysis of electromagnetic fields in an anisotropic material by a mapping theory.

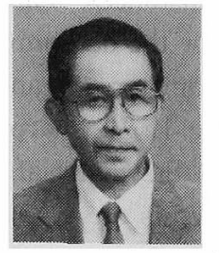

\section{Ryuiti Terakado (Member)}

He was born in Tokyo, Japan, on Jan. 30, 1930. He graduated from the Taga Technical College, Ibaraki, in 1950 . He received the D. E. degree from the Tokyo Institute of Technology, Tokyo, Japan, in 1975. He was a Professor during 1978 to 1989 , and Dean from 1985 to 1989 at the School of Engineering, Ibaraki University, Ibaraki, Japan. Since April in 1989, he has been the President of Fukushima College of Technology, Iwaki, Fukushima, Japan. His major research interests are two-dimensional fields and symmetry. 